\title{
Stochastic optimal energy management system for RTG cranes network using genetic algorithm and ensemble forecasts
}

Article

Accepted Version

Alasali, F., Haben, S. and Holderbaum, W. (2019) Stochastic optimal energy management system for RTG cranes network using genetic algorithm and ensemble forecasts. Journal of Energy Storage, 24. 100759. ISSN 2352-152X doi: https://doi.org/10.1016/j.est.2019.100759 Available at https://centaur.reading.ac.uk/84507/

It is advisable to refer to the publisher's version if you intend to cite from the work. See Guidance on citing.

To link to this article DOI: http://dx.doi.org/10.1016/j.est.2019.100759

Publisher: Elsevier

All outputs in CentAUR are protected by Intellectual Property Rights law, including copyright law. Copyright and IPR is retained by the creators or other copyright holders. Terms and conditions for use of this material are defined in the End User Agreement.

www.reading.ac.uk/centaur 
Central Archive at the University of Reading

Reading's research outputs online 


\title{
Stochastic Optimal Energy Management System for RTG Cranes Network Using Genetic Algorithm and Ensemble Forecasts.
}

\author{
Feras Alasali $^{\mathrm{a}, \mathrm{b}^{*}}$, Stephen Haben ${ }^{\mathrm{c}}$, William Holderbaum ${ }^{\mathrm{a}, \mathrm{d}}$ \\ ${ }^{a}$ School of Systems Engineering, University of Reading, Whiteknights, Reading RG6 6AY, UK; \\ ${ }^{\mathrm{b}}$ Department of Electrical Engineering, Hashemite University, Zarqa 13113, Jordan. \\ ${ }^{c}$ Mathematical Institute, University of Oxford, Andrew Wiles Building, Oxford OX2 6GG; \\ ${ }^{\mathrm{d}}$ School of Engineering, Metropolitan Manchester University, Manchester, UK.
}

\begin{abstract}
:
In low voltage networks, Energy Storage Systems (ESSs) play a significant role in increasing energy cost savings, peak reduction and energy efficiency whilst reinforcing the electrical network infrastructure. This paper presents a stochastic optimal management system based on a Genetic Algorithm (GA) for the control of an ESS equipped with a network of electrified Rubber Tyre Gantry (RTG) cranes. The stochastic management system aims to improve the reliability and economic performance, for given ESS parameters, of a network of cranes by taking into account the uncertainty in the RTGs electrical demand. A specific case study is presented using real operational data of the RTGs netwrok in the Port of Felixstowe, UK, and the results of the stochastic control system is compared to a standard set-point controller. In this paper, two forecast data sets with different levels of accuracy are used to investigate the impact of the crane demand forecast error in the proposed ESS control system. The results of the proposed control strategies indicate that the stochastic management system successfully increases the electric energy cost savings, the peak demand reductions and successfully outperforms a comparable set-point controller.
\end{abstract}

Keywords: energy storage system; RTG crane; genetic algorithm; stochastic control model; load forecast.

\section{Introduction}

\subsection{Background}

The trend in global consumerism and the increase in worldwide population requires improve merits in marine container transportation. According to trade statistical data from the World Shipping Council (WSC), 127.6 million twenty-foot container equivalent units (TEUs) were exported and imported globally in 2014 with a $4.3 \%$ increase from the previous year [1]. The United Nations report (Review of maritime transport, 2016) shows that the world gross domestic product expanded by $2.5 \%$ compared to 2014 and the world seaborne trade has expanded by $2.1 \%$ [2]. During the handling process of the container terminal, vast amounts of fuel are consumed and $\mathrm{CO}_{2}$ emissions produced, by the different handling and transfer equipment. Research carried out based on data released by the Port of Los Angeles to evaluate the gas emissions at container terminals, shows that $38 \%$ of the $\mathrm{CO}_{2}$ emissions comes from quay cranes and yard equipment such as RTG cranes and handlers. Furthermore, the $\mathrm{CO}_{2}$ emissions increased by $18 \%$ in 2014 compared to the previous year [3]. The high operating costs, pollution, and noise from the diesel yard equipment, has led sea ports to move towards replacing diesel RTG cranes with electric RTG cranes, which offer greater environmental friendliness and high energy efficiency. The use of electrified RTG cranes can increase the reduction in $\mathrm{CO}_{2}$ emission by $60 \%-80 \%$ [4]. Another benefit of replacing diesel RTG crane by electric ones, is the reduction in maintenance costs by around $30 \%$ [4]. As electricity demand is expected to increase due to the electrification of RTGs, terminal 
operators will need to meet this increase by reinforcing the electrical distribution network. In general, traditional solutions are focused on upgrading the existing power system infrastructure (e.g transformers and cables). These solutions are technically effective but commercially costly [4,5]. Energy Storage Systems (ESSs), the focus of this work, can be a significant solution to increase the energy efficiency of the port network system and decreasing environmental concerns. In this paper, the main role of using an energy storage device is to reduce the electric energy bill and RTGs peak demand by optimally shifting peak energy consumption to load demand periods. The network of cranes will require smarter control solutions to reinforce the electrical distribution networks and increase energy saving, where the behaviour of the RTGs electrical demand show a highly nonsmooth and volatile behaviour compared to low and medium voltage network demands. The energy forecasting and ESS solution roles are therefore expected to be more difficult [6,7].

\subsection{Literature review}

The conventional control strategies, such as set-point and (Proportional-Integral) PI controllers, that use a reference value of voltage [8,9], power [10] or State of Charge (SoC) [11], are used for individual RTG crane systems equipped with ESS and work by storing recovered potential energy. An ESS is charged when the crane lowers the containers and discharges during the lifting phase. However, the previous literature has not considered the volatility of the RTGs demand, the differentiation in the electricity prices or used a forecast profile to optimally control the storage device. This means the control implementations have a significantly limited performance. In addition, there is a lack of utilising optimal control methods in electrified RTG crane applications for reducing peak demand or increasing the energy cost savings. In their study, Pietrosanti et al. [5] introduced an optimal power controller for a diesel RTG with a storage device. The goal of the optimal controller was to operate a flywheel energy storage device under optimal operation conditions with uncertain stochastic power loads by charging and discharging the ESS during the lowering and lifting of the containers, respectively [5]. The simulation results were investigated over one-hour test cycles and showed that the optimal power controller achieved a peak reduction of $38.47 \%$ compared to $35.9 \%$ using a set-point controller. The optimal management controller in [5] assumed that the container weight was known in advance and did not target the daily peak demand on the potential energy cost savings based on the electricity price tariffs. In another study by Hellendoorn et al [12], an optimal algorithm was formulated for a diesel RTG crane equipped with

supercapacitor energy storage to reduce the fuel consumption. This controller was developed to determine the power output of the storage device in order to achieve the minimum fuel consumption. However, the proposed optimal controller required perfect future knowledge of the fuel costs and consumption during the operation crane cycle. The optimal control algorithm in [12] was exclusively developed for a diesel RTG crane and thus cannot be used with an electric crane systems. The results in [12] presented only the reduction in diesel consumption, and not peak power reduction. To the best of the author's knowledge, the reduction in electric energy cost and peak demand for electrified RTGs network has only been investigated in the authors previous work [7,13]. Alasali et al. [7] developed a Model Predictive Control (MPC) model and stochastic MPC [13], as an on-line receding horizon control solution. The MPC controller in [7] was used to control an ESS connected to single RTG system without considering the forecast model uncertainty or investigating the benefits of a central ESS for a network of RTG cranes. In [7], a rolling load forecast model was developed based on an Artificial Neural Network (ANN) method to provide the control system the future demand profile. The high uncertainty in the demand profile has a significant impact on the performance of the MPC energy storage control algorithms. 
The current literature in energy saving for LV network applications and microgrids has begun to investigate the benefits of incorporating the demand uncertainty by developing stochastic control methods in order to improve the performance of the ESS in the distribution networks [13]. An electrified RTG crane demand profile has highly stochastic and less predictable behaviour compared to other low voltage loads due to the lack of strong daily and annual seasonalities [6,7]. Challenges in developing accurate crane demand forecasts make it substantially more difficult to optimality control the ESS and increase the energy systems' efficiency using probabilistic forecasts.

In the literature MPC is used as a real time controller for RTG cranes [7,13] and electric vehicles [14] to improve the energy efficiency of the distribution network. In general, the real-time control presented in previous works [7,13] often relies on expensive communications and monitoring infrastructure being deployed. Therefore, this article will develop and present a stochastic optimal controller based on a Genetic Algorithm (GA), as an off-line optimal control solution, to incorporate the volatile demand nature and forecast model uncertainty to increase the benefits of using ESS in a network of RTG cranes. The off-line optimal controller reduces or eliminates the required communications infrastructure costs if the ESS schedule control can provide sufficient robustness to the storage benefit. In addition, the off-line optimal controller will reduce the need to have access to large quantities of demand and network data and controllable assets. This paper will investigate the potential of using an optimal off-line control algorithm based on a GA in an RTG cranes network equipped with a storage device. Furthermore, this work will present the impact of the forecast accuracy on the control model performance by using two different forecast profiles with wide differences of accuracy unlike previous studies [7,13], which have only considered a single forecast type.

A GA optimal controller has previously been used to effectively control an ESS in LV applications in order to increase energy savings [15,16]. For example, Pena-Bello et al. presented an optimisation operation algorithm based on a GA for battery storage systems in grid-connected housing with a PV system [15]. The optimal control model was used to generate a 24-hour optimisation battery framework that aimed to minimise the daily electricity bill by using a perfect day ahead forecast profile for the electricity, PV power output and electricity prices. The results show the significance of using forecast profiles to maximise the profit by combining PV and battery systems. Significantly, the model in [15] assumes a perfect forecast for the residential load and PV generation. In addition, the research did not consider the impact of the forecast error and the variability of a PV system on the operation model and results. Similarly, Mohamed and Koivo [16] presented a GA optimal operating strategy for a microgrid system to reduce the energy and gas emission costs and meet the required demand [16]. The proposed control model used the historical data and previous solution scenarios to improve the current control model performance. However, the energy management system response was affected by the weather conditions and actual demand load. Hence, by considering load and weather forecasts, the assessment of the energy management system in [16] can be improved. The previous literature has shown that a stochastic optimal management system based on a GA can be beneficial for the peak demand and electricity bill reduction at ports.

\subsection{Contributions}

The demand profile of a network of RTG cranes has less predictable behaviour compared to other electrical distribution applications such as residential or commercial customers [6]. In general, LV network demands have daily or seasonality patterns and high correlations with different exogenous parameters, such as 
temperature, which help to increase the demand prediction model accuracy. The non-smooth and volatile crane demand have no such obvious seasonalities, as well as ambiguous explanatory relationships with external variables. This increases the challenges in forecasting crane of demand and optimising the control of the ESS in a network of cranes compared to say, LV demands [6]. This paper attempts to fill the gap in the literature by developing a GA controller, as an off-line optimisation control system, for a cranes network equipped with a storage device to reduce the electric energy bill and peak demand compared to the more common controller in crane systems, set-point controller. This has significant international appeal due to the increased electrification of RTG cranes [3,4]. This article introduces a number of key contributions to the controlling of an ESS in an RTG cranes network.

- Firstly, we develop an off-line stochastic optimal controller, based on a GA that incorporates the uncertainty of the crane demand in order to minimise the peak demand and electric energy bill. This is in contrast to the limited literature that focuses on using standard control algorithms on diesel RTG crane systems. In addition, the stochastic optimal controller does not require any recent model information, or a rolling forecast model as presented in the authors previous work [13].

- Secondly, unlike previous studies [7,13], which only use an single forecast profile to feed the ESS control system and do not investigate the impact of the forecast accuracy on the control model performance, two types of forecast models of varied accuracy are compared to evaluate the proposed GA optimal controller and understand the impact of forecast errors on the energy storage system performance. Furthermore, the rolling forecast model in $[7,13]$ assumed knowing the container weight and number of crane moves in advance to achieve accurate forecast results, this paper investigates the ESS performance with a forecast model that does not require this assumption.

\subsection{Outline of paper}

The remaining of this article is presented as follows: the topology of the RTG crane network and the ESS systems are presented in Section 2. In the following section, the forecast model for RTG crane demand and the GA optimal controller are introduced. Then Section 4 presents and discusses the simulation results. Finally, a summary and conclusions of this paper are discussed in Section 5.

\section{RTG Cranes Network Demand and ESS Topology}

For port container terminals, the RTG crane is used to transfer containers in the yard area or onto trucks, as shown in Figure 1. Typically, most of electricity energy consumption in the crane system comes from lifting containers with various weights and different trajectories [5,7]. The electrical demand is typically increased when cranes move heavier containers [6,7]. In this article, the electrified RTGs, as shown in Figure 1, currently use at the Port of Felixstowe, UK, and are powered by a $11 \mathrm{KV} / 415 \mathrm{~V}$ transformer via a $217 \mathrm{~m}$ conductor bar system. These electrified RTG cranes have been manufactured by Shanghai Zhenhua Heavy Industries (ZPMC, Shanghai, China) [5,7].

In this work, two electrified RTGs network equipped with a central energy storage device is used, unlike the previous literature [7] which mainly focused on investigating the cost saving for a single crane model. Figure 2 shows the electrical single line diagram at the Port of Felixstowe, UK [13]. The ESS location in Figure 2 is motivated by the literature that used a central ESS in a LV substation for residential or commercial 
customers [17]. The ESS location close to the substation gives extra support to the secondary substation, mitigates operational constraints and can help to resolve thermal issues in this zone.

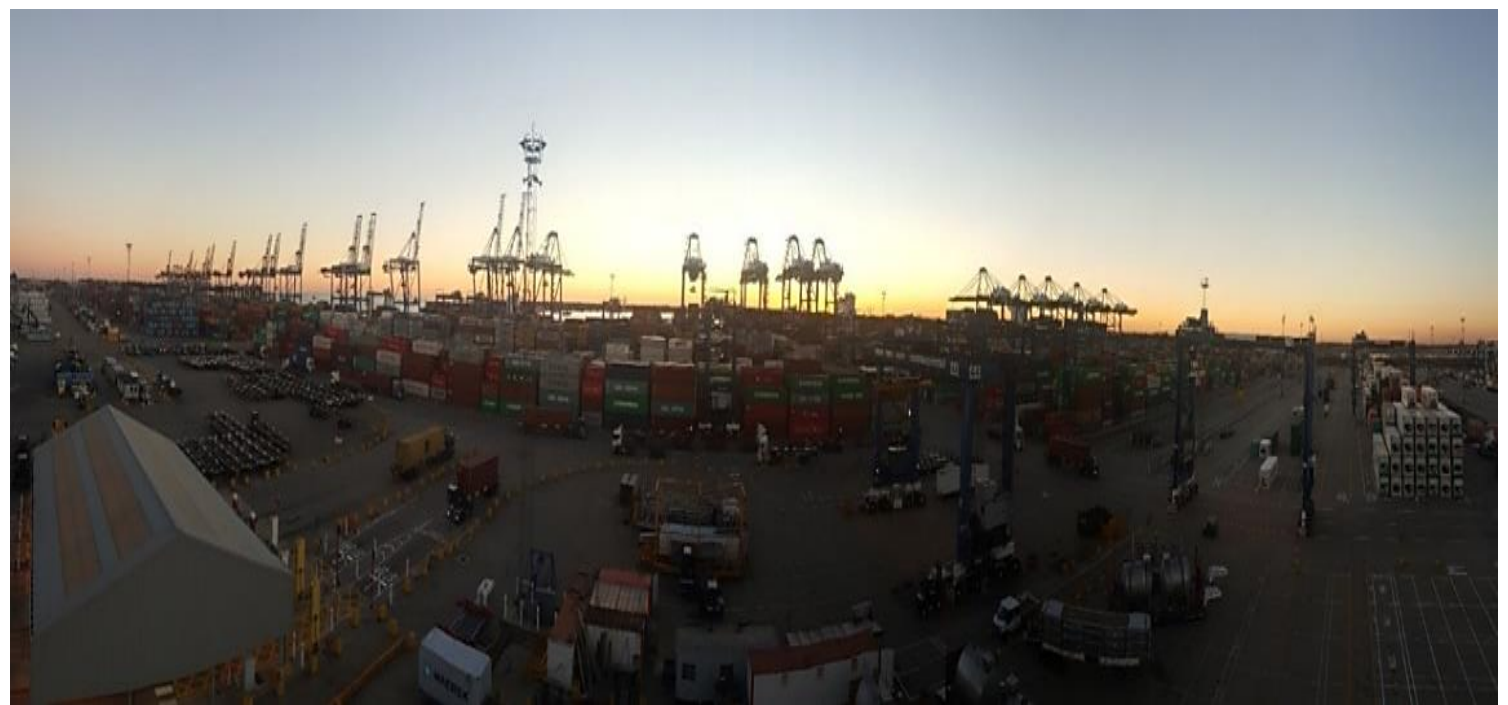

Figure 1. RTG cranes at the Port of Felixstowe (PoF), UK.

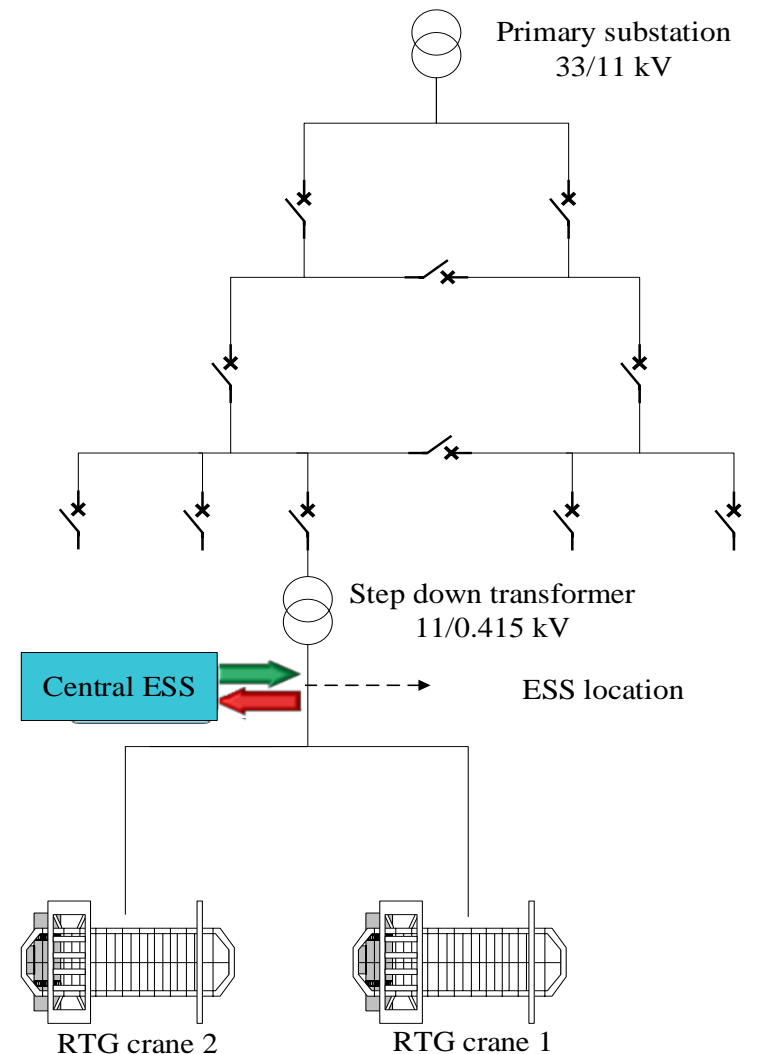

Figure 2. RTG cranes network including the ESS location scenario [13].

\subsection{The Energy Storage System (ESS) model}

The aggregation power sources, the secondary substation and the ESS, feeds the required demand consumption to run and operate the RTG cranes network.

Let 


$$
\mathrm{D}_{\mathrm{L}}=\left(\mathrm{D}_{\mathrm{L}}(1), \ldots, \mathrm{D}_{\mathrm{L}}(48 \mathrm{H})\right)^{\mathrm{T}} \in \mathbb{R}^{48 \mathrm{H}},
$$

be the complete half-hourly historical data set over $\mathrm{H}$ days. The ESS controllers are developed to find the optimal ESS operation plan over a day time period. Therefore, without loss of generality we focus on the half hourly crane demand for day $\mathrm{k}$, where $\mathrm{n}(\mathrm{n}=1,2, \ldots, 48)$ is the half-hourly time step over this day. Described as follows

$$
\mathrm{L}_{\mathrm{k}}(\mathrm{n})=\mathrm{D}_{\mathrm{L}}(\mathrm{n}+(\mathrm{k}-1) 48) \in \mathbb{R}_{\geq 0}^{48},
$$

The research objective here is to maximise the electric energy cost savings and minimise the peak demand using an energy storage device connected to the crane network. Equation (3) simulates the RTGs network demand equipped with the storage device.

$$
\mathrm{S}(\mathrm{n})=\left(\sum_{\mathrm{i}=1}^{\mathrm{c}} \mathrm{L}_{\mathrm{k}}^{(\mathrm{i})}(\mathrm{n})\right)+(\Delta \mathrm{E}(\mathrm{n}))
$$

where $S(n)$ is the secondary substation demand at time $n, \sum_{i=1}^{c} L_{k}^{(i)}$ is the total of the RTGs electrical demand $\left(\mathrm{L}_{\mathrm{k}}=\left(\mathrm{L}_{\mathrm{k}}^{(1)}, \ldots, \mathrm{L}_{\mathrm{k}}^{(\mathrm{c})}\right) \in \mathbb{R}^{\mathrm{c}}\right), \mathrm{c}=2$ is the number of RTGs and $\Delta \mathrm{E}(\mathrm{n})$ is the charging or discharging energy for the storage device at time $\mathrm{n}\left(\Delta \mathrm{E}=(\Delta \mathrm{E}(1), \Delta \mathrm{E}(2) \ldots, \Delta \mathrm{E}(48))^{T} \in \mathbb{R}^{48}\right)$. The ESS model presented in this paper has been used in the literature [7,13,17] and has constraints described by below Equations (4) - (7). Typically, The State of Charge (SoC) of the ESS at the time step n is SoC(n). The SoC represent the stored energy in the storage device and depends on the previous $\operatorname{SoC}$ value, $\operatorname{SoC}(n-1)$, and $\Delta E(n)$ as described by Equation (4).

$$
\operatorname{SoC}(\mathrm{n})=\operatorname{SoC}(\mathrm{n}-1)+\tilde{\theta} \cdot \Delta \mathrm{E}(\mathrm{n})
$$

The ESS model considers the efficiency of the device by combining the $\Delta \mathrm{E}(\mathrm{n})$ into a variable representing the ESS efficiency, $\tilde{\theta} \in[0,1]$. The efficiency of the ESS is related to the ESS operation mode, charging or discharging, as described by Equation (7). The operation of the ESS is subject to a number of constraints: the maximum and minimum stored energy SoC ${ }^{\min }$ and $\mathrm{SoC}^{\max }$, respectively, and the upper and lower limit of the changes in stored energy, $\Delta \mathrm{E}^{\mathrm{min}}$ and $\Delta \mathrm{E}^{\mathrm{max}}$, respectively, described by Equations (5)-(7) [7,13].

$$
\begin{aligned}
& \left.\begin{array}{c}
\mathrm{SoC}^{\min } \leq \mathrm{SoC}(\mathrm{n}) \leq \mathrm{SOC}^{\max } \\
\Delta \mathrm{E}^{\min } \leq \Delta \mathrm{E}(\mathrm{n}) \leq \Delta \mathrm{E}^{\max }
\end{array}\right\}, \quad \forall \mathrm{n} . \\
& \tilde{\theta}=\left\{\begin{array}{l}
\tilde{\theta} \text { if } \Delta E \geq 0 \\
\frac{1}{\tilde{\theta}} \text { if } \Delta E<0
\end{array}\right.
\end{aligned}
$$

In this paper, the optimal controller is used to determine an optimal changing value of the stored energy, $\Delta \mathrm{E}(\mathrm{n})$, that maximises the energy savings and reduces the peak demand. $\Delta \mathrm{E}(\mathrm{n})$ is increased (charging mode) and decreased (discharging mode) based on the RTGs electrical demand, $\mathrm{L}_{\mathrm{k}}(\mathrm{n})$, and ESS operation limitations. The $\Delta \mathrm{E}$ can have a positive/negative value to present the charging/discharging mode in the ESS and is described as $\Delta \mathrm{E}(\mathrm{n})=\operatorname{SoC}(\mathrm{n})-\operatorname{SoC}(\mathrm{n}-1)$. Generally, the storage device control is presented by the average power term, $\mathrm{P}_{\mathrm{E}}$ in $\mathrm{kW}$, and the stored energy term as $\mathrm{P}_{\mathrm{E}} \Delta$ time in $\mathrm{kWh}$, where $\Delta$ time represents the time period (usually hours) [7,13,17]. 


\section{Stochastic Optimal Energy Controller.}

In the literature, MPC controller systems are implemented using a rolling point forecast model [7]. However, in realistic scenarios the demand has different level of uncertainty. This is particularly true for RTG crane, where even when the crane is lifting the same container weight, the demand is quite variable [6,7]. Probabilistic models are required, which can model these uncertainties. Here a stochastic optimal energy management is developed which is more efficient at increasing the cost saving and the peak demand reduction. The stochastic model aims to include the diversity of the RTG cranes demand and the forecast error. To solve the stochastic energy optimisation under the uncertainties problem in the forecast of RTGs demand, this article introduces a stochastic optimisation management system using a Genetic Algorithm (GA). The main outline scheme of the ESS stochastic optimal controller is shown in Figure 3. Firstly, the RTG cranes data is collected and analysed. Secondly, an ensemble forecast model is developed based on a Monte Carlo and Autoregressive Integrated Moving Average with Explanatory Variable (ARIMAX) to create a number of day ahead RTGs electrical demand scenarios [13]. Then, the Genetic Algorithm (GA) is designed to find the ESS optimal operation schedule over the ensemble of demand profiles.

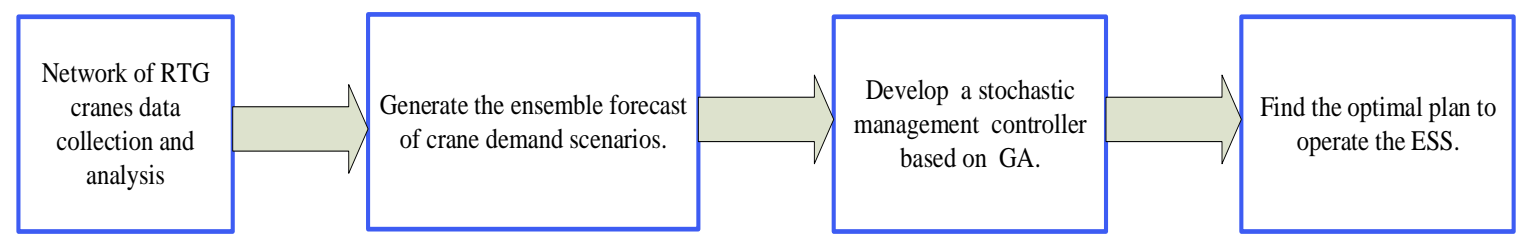

Figure 3. General schematic of the proposed stochastic optimal controller.

\subsection{Electrified RTG cranes data.}

The smart meter data for two RTGs, at the Port of Felixstowe in the UK, were collected over the period $1^{\text {st }}$ to $30^{\text {th }}$ of April 2017. This port runs 85 RTG cranes continuously up to 24 hours daily over the year to handle and operate more than 4 million TEUs every year [18]. The collected data at a half hour resolution (the RTGs electrical demand, number of cranes moves and container weight) describes the real daily crane behaviour at ports. The first $80 \%$ of the crane data is used to train and validate the forecast and ESS control models (25 days of data), and the final $20 \%$ of training data is used to evaluate the ESS performance ( 5 days). Furthermore, due to the volatile and non-smooth nature of the RTGs electrical demand and the lack of seasonality or trends over the time series, a longer time series data will likely have negligible effect on the ESS control and forecast model results. The number of simulations generated in this paper for different forecast profile scenarios including the varying characteristics of the crane demand cover the normal operation days and is similar to those given in the literature $[6,7,13]$.

\subsection{Ensemble forecast model}

To develop an optimal controller, the expected future energy demand must be estimated. The future RTGs electrical demand for a day ahead, $\hat{\mathrm{L}}_{\mathrm{k}}$, is presented and modelled in this section probabilistically by generating $\mathrm{W}$ of possible future demand profiles by using an ensemble forecast model (ARIMAX and Monte Carlo) [13]. The half hourly future demand of day $\mathrm{k}$ for ensemble $\mathrm{w}$, where $\mathrm{w} \in\{1, \ldots, \mathrm{W}\}$ is written as: 


$$
\widehat{\mathrm{L}}_{\mathrm{k}}{ }^{\mathrm{w}}=\left(\widehat{\mathrm{L}}_{\mathrm{K}}{ }^{\mathrm{w}}(1), \hat{\mathrm{L}}_{\mathrm{K}}^{\mathrm{w}}(2) \ldots, \hat{\mathrm{L}}_{\mathrm{k}}{ }^{\mathrm{w}}(48)\right)^{\mathrm{T}} \in \mathbb{R}^{48}
$$

The electrified RTGs electrical demand forecast procedure is autonomously trained prior to the development of the ESS control model. The ARIMAX forecasts for a single RTG crane has been previously developed by the authors [6,13]. To present the volatile and uncertain crane demand, the ARIMAX forecast model [6] has been modified to create several potential RTG crane demand forecast scenarios by using a Monte Carlo sampling method. Here, we sample the crane demand from the joint probability distribution for the RTG electrical demand and container gross weight. Then, the ARIMAX is used to obtain the forecast time series output responses. Unlike the previous study [7,13], that using a rolling ANN forecast model (a relatively accurate forecast model) with average Mean Absolute Percentage Error (MAPE) equal to 14\% over 5 days. The modified ARIMAX model coupled with using the Monte Carlo model will model the uncertainty term by generating the future crane demand scenarios from two types of forecast models, with varied accuracy, and without updating the forecast model within the day. This modifys the following two forecast models that were developed in [6]:

- Accurate forecasts: the future demand estimates are generated by using the most accurate forecast model (ARIMAX), Model C.2 as presented in [6], which only estimates the number of the RTG moves while assuming the weight of containers are known, in advance. This forecast has errors between $14 \%$ and $28 \%$.

- Inaccurate forecasts: the future demand estimates are generated by using a relatively inaccurate forecast model (ARIMA) that does not include any of the above exogenous variable data, Model F as presented in [6]. This gives a MAPE between $27 \%$ and $40 \%$.

The two types of forecast data sets have been used to evaluate the proposed optimal controller with different forecast profiles to understand the impact of forecast errors on the energy storage control algorithms.

\subsection{Stochastic optimal energy management system using GA.}

The genetic algorithm is an iterative optimisation technique for solving complex problems by considering several different possible solutions in each iteration [19]. The GA methodology is inspired by the natural evolution process of living organisms [20]. In energy systems, the GA has been applied to solve complex energy optimisation problems in many different applications [21-23]. However, these studies typically only use GA to solve optimisations which utilise a single demand scenario.

The GA operates on a fixed population size of possible solutions, called chromosomes or individuals. During the implementation process, all solution of the current generation are evaluated by a fitness function. The fitness of each possible solution is directly related to the optimization problem via a fitness or cost function. The GA employees three main operations to produce a new population: selection, crossover and mutation [19,20]. Figure 4 presents the general flowchart of the standard GA. The GA process starts with a set of possible solutions. Then, using an evaluation of the fitness of each solution and an appropriate selection technique, parent solutions are picked to create a population of new offspring (generation). The GA process is repeated until a maximum number of generations or threshold is reached. The GA performance mainly depends on the reproduction step which involves crossover and mutation operation. Selecting the suitable population size, crossover and mutation are essentially to maintain the diversity in the population and guide the optimal search process [24-26]. 


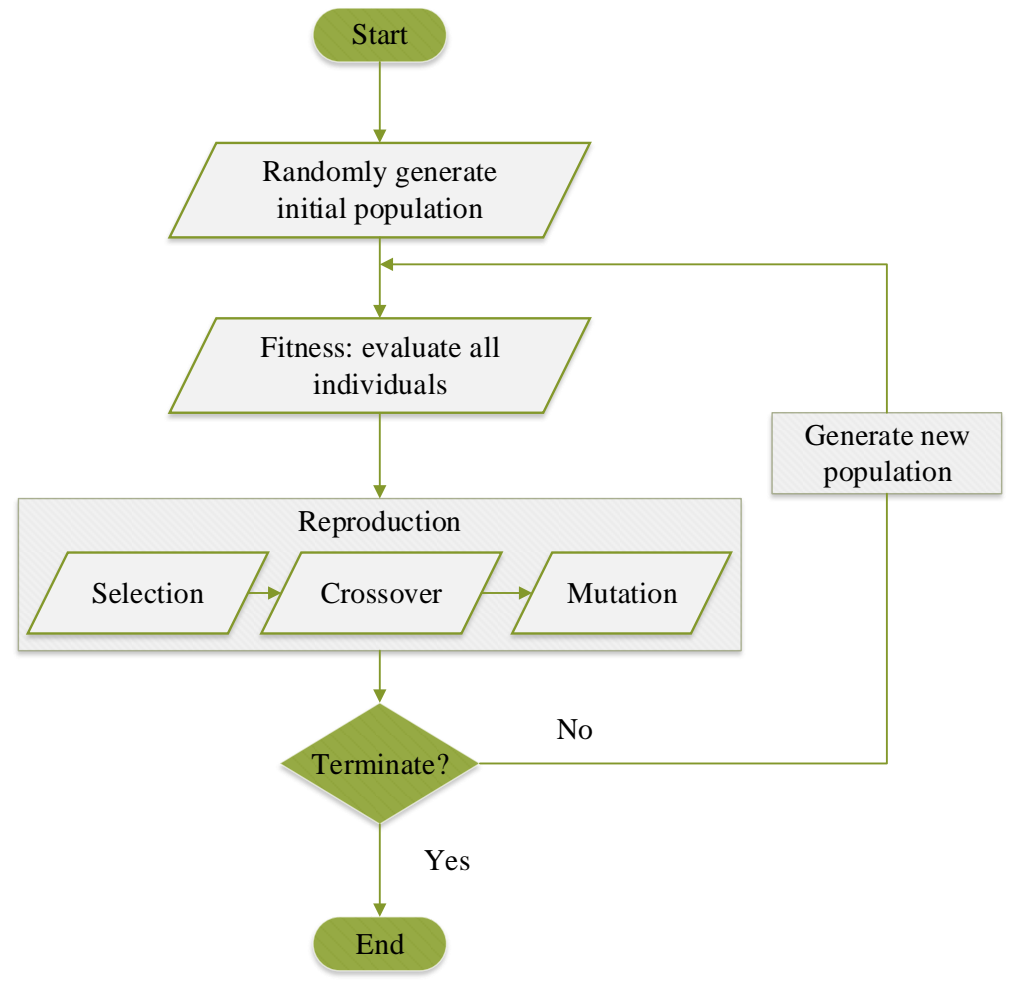

Figure 4. Genetic algorithm flowchart.

\subsubsection{Stochastic optimisation controller for RTG crane demand}

In this work, a genetic algorithm (GA) is developed to optimise the peak demand electricity bill for a network of RTGs connected to an ESS, as seen in Figure 5. The RTGs electrical demand uncertainty is treated in this study by generating a number of possible future demand scenarios, as discussed in Section 3.2. Suppose we have a W forecast of demand ensembles for the RTG crane, labelled $\widehat{\mathrm{L}}_{\mathrm{k}}{ }^{\mathrm{w}}$ where $\mathrm{w}=1, \ldots, \mathrm{W}$, is described by Equation (8). The control model aims to operate the ESS by determining the optimal $\triangle \mathrm{E}(\mathrm{n})$ over the prediction period. At the start of the GA process, as seen in Figure 5, an initial population of ESS operation profiles is generated and evaluated by a feasibility test (described in detail later). Assuming the generation of M ESS profiles where $\mathrm{j}=1, \ldots, \mathrm{M}$, we let $\mathcal{P}$ be the set of all profiles, $\Delta \mathrm{E}^{\mathrm{j}}$, as described by,

$$
\mathcal{P}=\left\{\Delta \mathrm{E}^{\mathrm{j}}=\left(\Delta \mathrm{E}^{\mathrm{j}}(1), \ldots, \Delta \mathrm{E}^{\mathrm{j}}(\mathrm{N})\right)^{\mathrm{T}} \in \mathbb{R}^{\mathrm{N}}, \mathrm{N}=48\right\} .
$$

The control method, in this section, searches for a half hourly ESS profile $\Delta \mathrm{E}_{S}{ }^{j} \in \mathcal{P}$ to reduce the RTGs electricity bill and peak demand for the RTGs stochastic demand, $\widehat{L}_{K}$. The GA proceeds by creating a new ESS profiles to solve the optimisation problem to the storage profile performance according to a "fitness function" and the reproduction process (crossover and mutation). The GA method for the ESS is summarised and outlined below: 


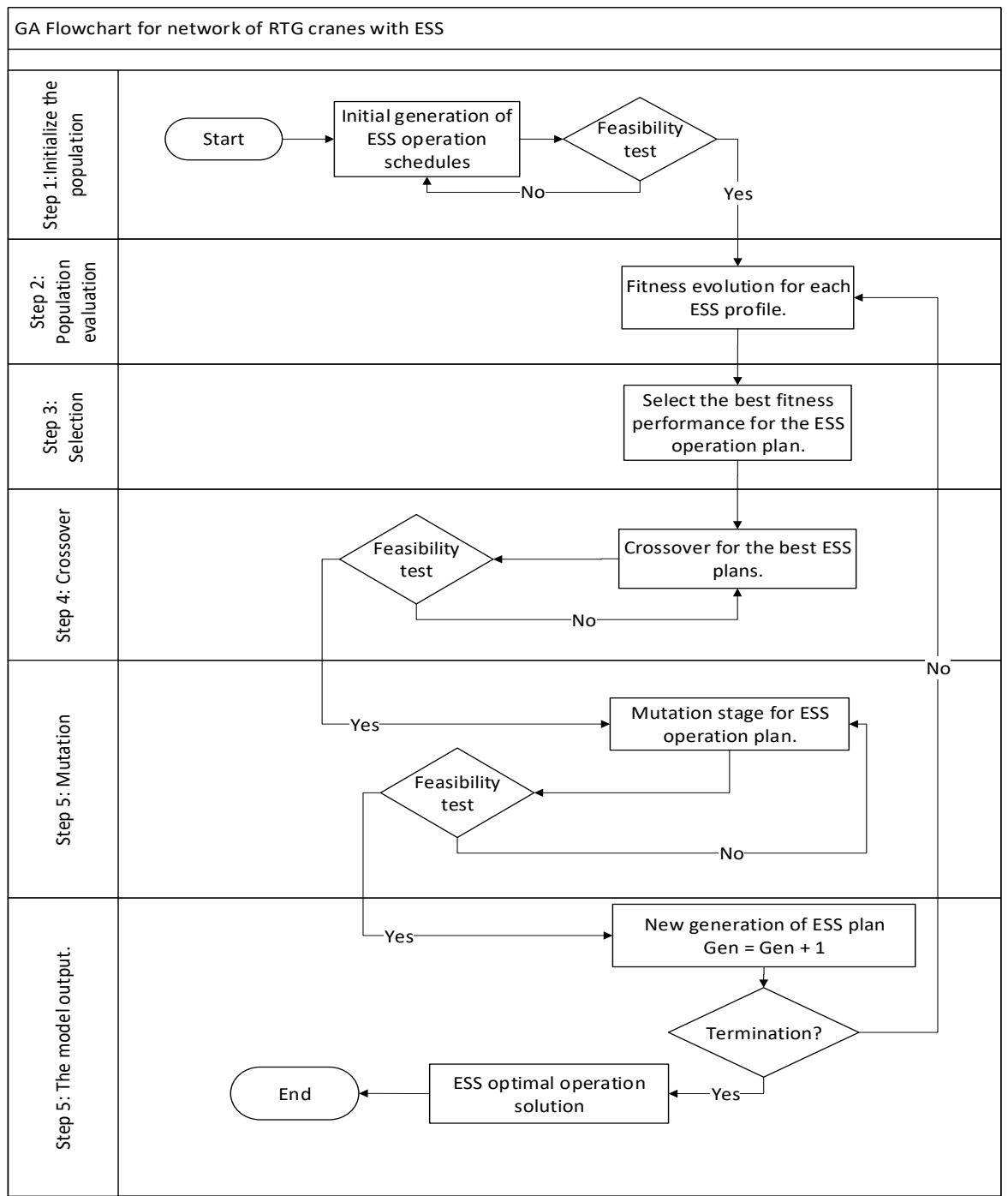

Figure 5. The ESS optimisation process of developed GA for RTG crane system.

Step 1 population initialization: create a number of random ESS profiles $\Delta \mathrm{E}^{\mathrm{j}}$, to apply on each future RTG demand profile, $\hat{\mathrm{L}}_{\mathrm{k}}{ }^{\mathrm{w}}$. The number of demand scenarios is chosen to provide a good balance between the performance and computational costs. The major challenge with choosing a random initialisation for the genetic algorithm is the requirement for the profiles to satisfy the crane model and ESS operation constraints, as presented in Section 2.1. The random profiles may violate these constraints and will increase the processing time. Therefore, the feasibility test in this step aims to make sure that all ESS profiles $\Delta \mathrm{E}^{\mathrm{j}}$ satisfy the crane model and ESS operation constraints. Unfeasible ESS profiles are rejected and another storage operation profile is randomly generated until the initial ESS profiles population is completed and feasible. The population size of 1000 is selected which is also similar to the literature $[15,16]$. Furthermore, based on the time resolution and design of the stochastic optimal controller, as an off-line controller, the computational cost was not excessive for the research proposed here. The proposed controller is designed to use a fixed forecast model with a daily updating scheme, unlike the previous work [7,13] that used a rolling forecast with real time measurement to update the forecast model every time step which is computationally expensive compared to the daily forecast model. In addition, the MPC and SMPC [7,13] controller required recent model information to recalculate the 
ESS control sequence at every time step which lead to an increase in the computational time and generated further computational constraints compared to the proposed control model in this paper.

Step 2: population evaluation, the fitness of each ESS profile is evaluated using a cost objective function. In this section, the cost function, as described in Equation (10) is daily electricity energy costs.

$$
E P^{w, j}=\sum_{n=1}^{N}\left(B(n)\left(\widehat{L}_{k}{ }^{w}(n)+\Delta E^{j}(n)\right)\right)^{2},
$$

where $B(n)$ is the electric energy cost at the time step (n) and $N$ is one day time steps $(N=48)$. In this work, we target the minimum energy costs, therefore, the fitness function is formulated as follows:

$$
\mathrm{F}^{\mathrm{w}, \mathrm{j}}=\frac{1}{1+\mathrm{EP}^{\mathrm{w}, \mathrm{j}}}
$$

This work aims to find the best possible energy storage plan for the stochastic RTG crane demand. Therefore, the fitness of the $\mathrm{j}^{\text {th }}$ ESS operation plan is formulated as in Equation (12).

$$
F^{j}=11+w=1 W E P^{w, j}
$$

Step 3: In order to select the best performing (fittest), ESS schedule a probability is assigned based on the fitness as defined in Equation (13). The parents for the next GA step are selected based on this probability. The fittest (best performing) ESS profiles are therefore more likely to be selected in the next generation.

$$
\text { Probability of } j=\frac{F^{j}}{\sum_{j=1}^{M} F^{j}} \text {. }
$$

Step 4: To generate of $\Delta \mathrm{E}_{\mathrm{S}}{ }^{\mathrm{j}}$ for the next level, the best (higher probability) two ESS plans are selected for crossover. The common genes of parent profiles are retained while the rest are determined randomly from the parents. The crossover process for the storage operation plan may violate the crane model and ESS constraint. Therefore, the feasibility test works to make sure that the new child of the ESS profile $\Delta \mathrm{E}^{\mathrm{j}}$ satisfies these constraints. The unfeasible child is rejected and another one is generated by retaining the common genes and randomly selecting the remaining genes until the crossover is feasible. At the early iterations, parent profiles are expected to be diverse and child profile may be distant from them. However, in final generations, the parent and child profiles become more similar and the search converges toward local optimal solution.

Step 5: The new child (ESS plan) from the previous step is mutated through replacing each gene with a probability $U$ by new random genes. As discussed previously, this process needs to follow the crane and ESS constraint. The unfeasible profile is rejected and another one is generated until the mutation process is completed. In this work, the probability $U$ is initially set to 0.1 and as the GA process and search converge toward a solution the probability slowly decreases [23,24].

Step 6: Repeat steps 1 to 3 for 200 iterations. 
An advantage of the genetic algorithm method is that it can easily include ensemble of RTG crane demands, which will help to generate an optimal ESS for different demand scenarios including worst case scenarios.

\section{Results and Discussions}

The results of running the off-line control algorithm (the stochastic optimization control based on GA) and setpoint controller for RTGs network connected to an ESS is presented and compared in this section. First, the forecast results are discussed; then the ESS controllers are tested. Throughout this subsection, the comparison results will introduce the peak reduction and electric energy cost saving for a specific case study using the following configurations:

- An ESS controlling by a set-point method as standard control model (widely used in the literature $[5,7,10])$. This controller compares the RTGs electrical demand to a reference value. This set-point value is predetermined by using historical RTGs demand. The ESS will be in charging mode when the demand is under the reference value and in discharging mode if the RTGs electrical demand exceeds the set-point. Furthermore, we take into account the electric energy tariffs to control the storage device and reduce the electricity bill by encouraging the storage to charge and discharge during the low and high tariff periods, respectively. The literature introduces the set-point model as standard and a simple controller for an ESS, but is principally limited especially when used on demand profiles with high uncertainty, as it takes the charge and discharge decisions without any future knowledge.

- An ideal ESS model based on a set-point control with infinite energy capacity; no limitations or constraints are imposed on the ESS in order to provide the greatest possible peak demand reduction. In this model, the set-point value will be the average half hourly demand of the day.

- Energy storage using a stochastic optimal controller based on GA is developed to maximise the benefits of using ESS compared to the benchmark controller. The GA control model has been evaluated by using two different forecast methods, as described in Section 3.2.

\subsection{Ensemble forecast results.}

The aim of developing a stochastic forecast model is to replicate the uncertainty in the RTGs electrical demand by generating a number of future demand scenarios to feed the optimal ESS control model. In Figure 6, the simulated ensembles from the ARIMAX and Monte Carlo model is presented. The scenarios of the future crane demand are shown in gray lines deviating mainly close to the actual RTGs electrical demand profile in every time step, showing the forecast has generally captured the uncertainty.

The forecast models in this paper feed the optimal control, different future demand scenarios. Generally, point forecasting [7] evaluation is usually based on a prediction error such as MAPE [27] and the interpretation of forecast error generating by ensemble forecasts is considered with a curve as presented in Figure 6. Ensemble forecast is common technique to generate the future demand scenarios [27] but due to the difficulties to compare ensemble forecast scenarios accuracy to point forecasting models, the mean value of all scenarios has been used to generate a comparison in Section 4.1. Overall, the ensemble forecast and point forecast [7] aren't directly comparable and the comparison cannot deliver a decisive conclusion but can show the significant of using different forecast method to improve the ESS performance. In addition, unlike the previous work $[7,13]$ that 
assumed knowing the container weight and number of crane moves in advance to achieve accurate forecast results, this paper investigates the ESS performance with an inaccurate forecast model that does not require this data. In Table 1, the rolling forecast process in [7,13] decreased the average MAPE by approximately $8 \%$ to $10 \%$ compared to the proposed accurate forecast model in this paper with daily updating. The high MAPE doesn't mean that the forecasts are not appropriate for the very different control mechanism where it is important to handle the uncertainty in the prediction profile by using ensemble forecast to improve the storage performance. In addition, the rolling forecast required a real time measurement in order to update the forecast model every time step and is computationally expensive compared to the daily forecast model. In addition, unlike the previous work [7,13] that assumed knowing the container weight and number of crane moves in advance to achieve accurate forecast results, this paper investigates the ESS performance with an inaccurate forecast model that does not require these data.

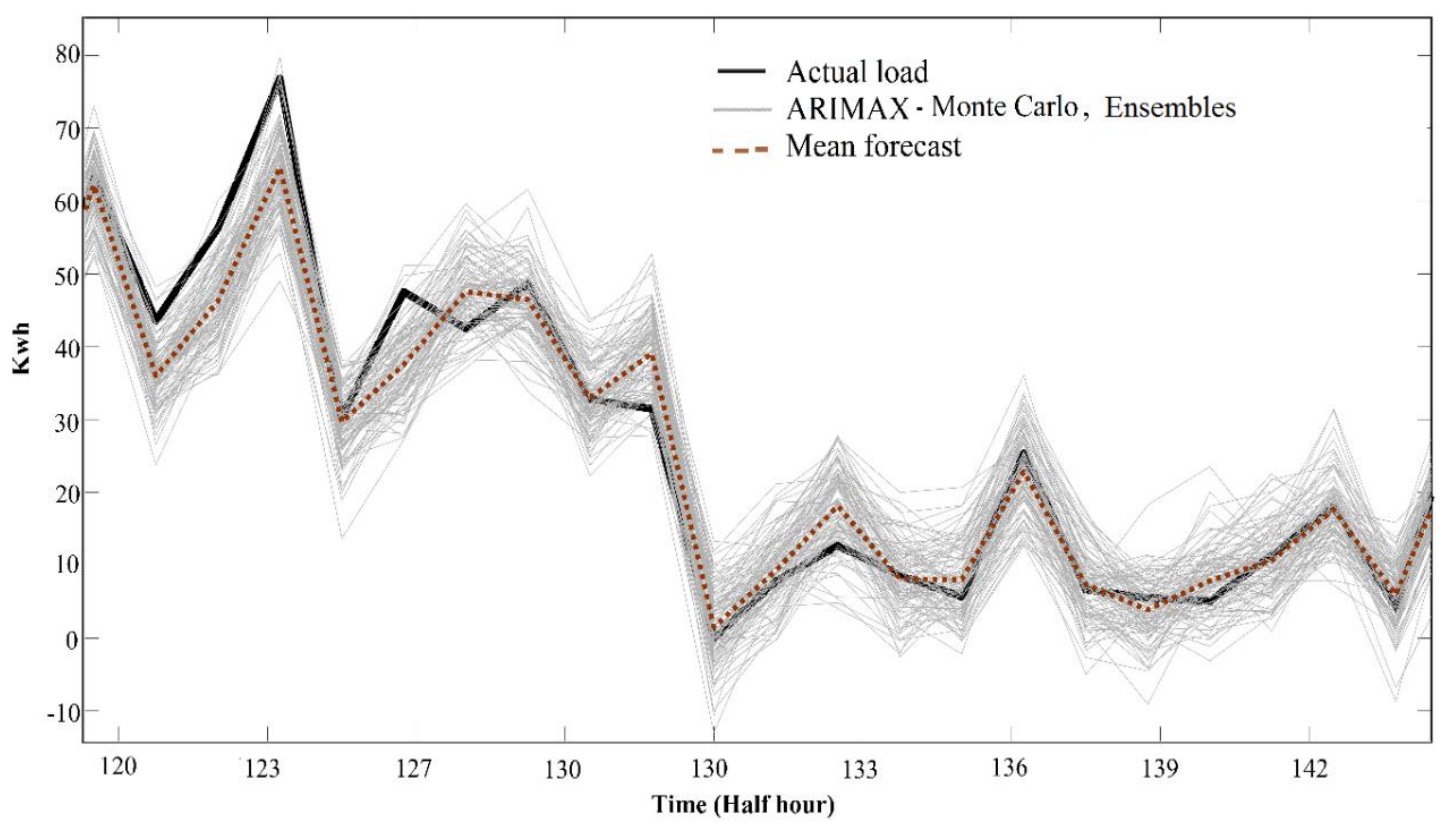

Figure 6. Example of the ensemble forecasts.

Table 1. The average of the daily MAPE for the mean of cranes demand forecast scenarios: ANN [7], ARIMAX [13], ARIMA and fixed ARIMAX prediction models.

\begin{tabular}{cc}
\hline Forecast model & MAPE \\
\hline ARIMA (inaccurate forecast model) & $30.1 \%$ \\
ARIMAX (Accurate forecast model) & $22.4 \%$ \\
Rolling ARIMAX model [13] & $12.6 \%$ \\
Rolling ANN model [7] & $14.3 \%$ \\
\hline
\end{tabular}

\subsection{Stochastic optimal control (GA) results for a specific case study.}

The GA optimal management controller is implemented in a RTGs network connected to a single storage device. The collected data, as described in Section 3.1, and ESS parameters, as presented in Table 2, were used to evaluate the ESS performance controlling by the stochastic GA and the set-point model. The ESS parameters 
in Table 2 is similar to the literature [7,17]. Hong-lei et al. have been developed a lithium battery energy system with capacity $128 \mathrm{kWh}$ and SoC from $0 \%$ to $100 \%$ for a hybrid operation RTG crane [28]. Furthermore, the Vahle company proposed two ESS sizes as follow: (a) $80 \mathrm{kWh}$ battery system for a hybrid RTG crane system, (b) $120 \mathrm{kWh}$ battery energy storage device for peak reduction and also to allow the electrified crane to work a few hours independently from LV network [29,30]. The ESS size (80 and $120 \mathrm{kWh}$ ) presented by the Vahle company for an RTG system is basically equal to $9.8 \%$ or $14 \%$ of the maximum daily, over an 8 days period, RTG electrical demand in [30], the ESS size was chosen based on the ESS role in this paper and between the above storage sizes (approximately $10.5 \%$ of the maximum daily RTG demand) [31]. In general, a small ESS size rises the likelihood of missing critical peak demand due to the energy storage limitation. In addition, Table 3 introduces the parameters of the GA controller, as discussed in Section 3.3.

Table 2. The main parameters of the ESS in RTGs network.

\begin{tabular}{cc}
\hline Parameter & Value \\
\hline SoC & $10 \mathrm{kWh}$ \\
SoC & $170 \mathrm{kWh}$ \\
$\Delta \mathrm{E}^{\min }, \Delta \mathrm{E}^{\max }$ & $\pm 170 \mathrm{kWh}$ \\
\hline
\end{tabular}

Table 3. The parameters of the GA model.

\begin{tabular}{cc}
\hline Parameter & Value \\
\hline Population size & 1000 \\
Number of iterations & 200 \\
Mutation & 0.1 \\
Demand scenarios & 50 \\
\hline
\end{tabular}

\subsubsection{Peak demand reduction}

Table 4 presents the forecast models that have been used to implement the ESS control models, as described in Section 3.2. To better understand the stability and robustness of an ESS storage control technique for a network of cranes, we also consider the effect of the forecast error on the performance of an ESS. In the literature, ESS controllers for LV network applications typically use perfect forecast models [13,32] or forecasts of systems with relatively low errors between $10 \%$ to $15 \%$ [17,33]. Therefore, in this section, two types of forecast models, accurate and inaccurate, have been used to evaluate the proposed GA controller and understand the impact of the forecast errors on the energy storage control performance.

The ESS control algorithms results, shown in Table 4, demonstrate that the accurate forecast models help the optimal controller to improve the energy storage performance and increase the peak demand reduction compared to optimal controller with inaccurate forecasts. For illustration, the percentage of peak reduction decreased to $24.1 \%$ from $27.7 \%$ for the GA control model. The stochastic algorithms allow the control model to investigate and evaluate the ESS over a number of demand profile scenarios, which helps to reduce the impact 
of a high forecast error. Figure 7 presents the relationship between the forecast errors and peak reduction for the GA controller over the testing data period. These results shown that a more accurate forecasting method can help to achieve greater peak reduction. The accuracy of the forecast model is a significant guide for potential peak reduction in the optimal controllers and more accurate forecast likely indicate a large have good peak reduction [34]. However, the more accurate forecast do not always guarantee a greatest peak reduction over all the time points, as seen in Figure 7, since the forecast error could be concentrated of one peak point rather than to be distributed over the day time which leads to increases in the peak. However, the loss in performance due to forecast error is no very large as seen in Table 4.

Table 4. The average peak demand reduction for the GA controller with average forecast errors.

\begin{tabular}{ccc}
\hline Type of the forecast model & \%MAPE & \% Peak reduction \\
\hline Accurate forecast model. & $22.8 \%$ & $27.7 \%$ \\
Inaccurate forecast model & $34.1 \%$ & $24.1 \%$ \\
\hline
\end{tabular}

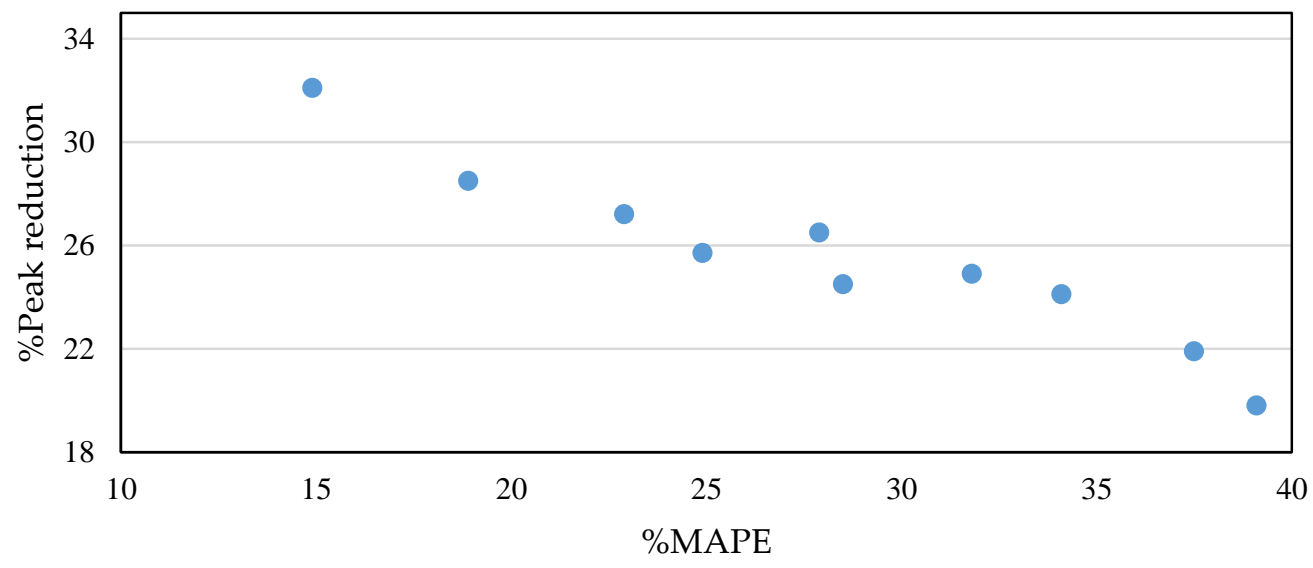

Figure 7. The relationship between MAPE and daily percentage of peak reduction for GA controller for accurate forecast model.

The GA optimal controller use RTGs prediction demand profile to create an optimal charging and discharging schedule for the ESS, considering the electric energy cost rate and the energy storage limitation, as described in Section 2.1. Due to the volatile and stochastic nature of the RTGs electrical demand and forecast errors, the ESS is unable to generate the highest possible RTGs peak demand reduction as could be achieved with a perfect future demand knowledge, as seen in Figure 8. The GA controller outperforms the set-point controller. The GA controller with accurate forecast achieved a $27.7 \%$ peak demand reduction compared to $23.9 \%$ for set-point. Using an inaccurate forecast model for running the controller, the GA optimal controller also outperformed the set-point model. The ideal ESS model with infinite capacity and no limitations, basically producing a flat profile, reached a reduction of $64.8 \%$, and this is the highest possible peak reduction in the crane demand. In general, the reduction in RTGs peak electrical demand level will help also to reduce the stress, thermal problems, on the port's electrical infrastructures and increase cost saving, except for the ideal ESS model where 
the cost constraints cannot be included in this model. In the following section, we will consider the electric energy cost and present the cost savings for a network for cranes.

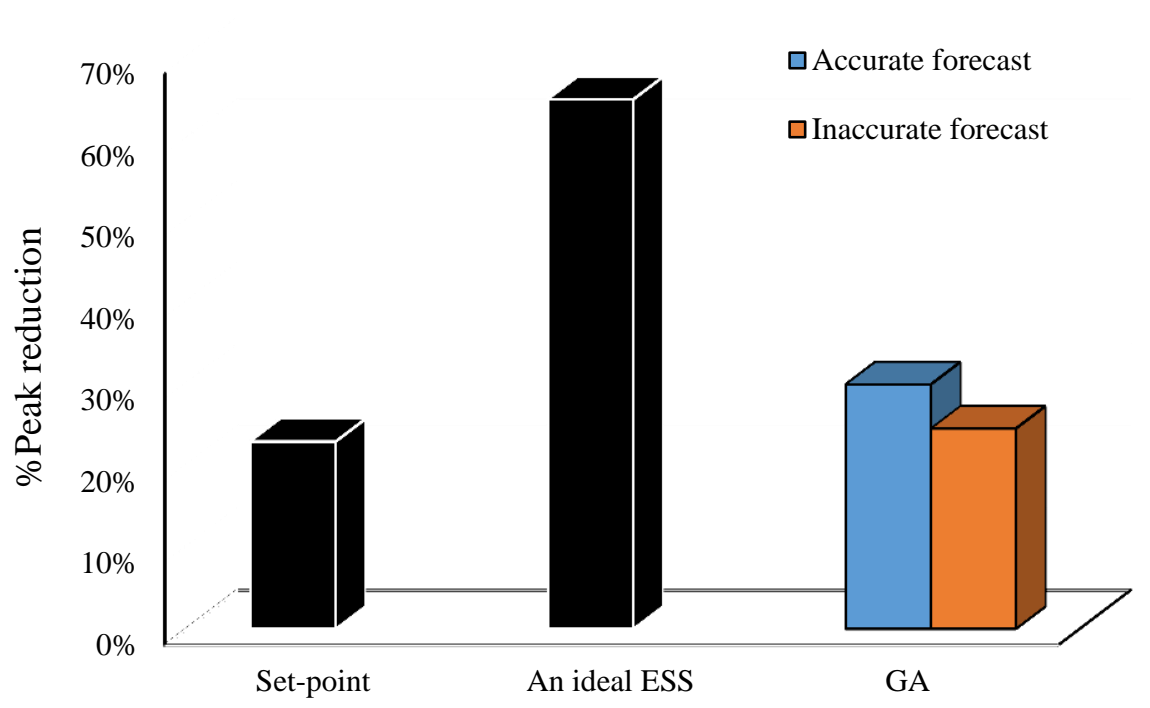

Figure 8 . The average percentage of peak demand reduction results.

As previously discussed, the cranes network demand is non-smooth and less predictable compared to medium or low voltage demands such as household, electric vehicle and renewable energy [6,13]. Therefore, the cranes demand and medium or low voltage demands aren't directly comparable due to the different demand nature. The following peak reduction results from the current literature aim to provide illustrative examples and show the significance of using the ESS with different systems. M. Rahmani-Andebili presented a $19.7 \%$ energy loss reduction by using MPC [35] and 23\% cost saving by using a stochastic MPC considering different forecast errors [36] for ESS in a medium distribution network with renewable. The simulation results of M. RahmaniAndebili and Fotuhi-Firuzabad study [14], showed approximately $15 \%$ of energy cost saving by using a GA optimisation in SMPC, for a charging management system for plug-in electric vehicles in a low voltage network.

\subsubsection{Economic benefits of using ESS in RTGs network.}

In this paper, the GA controller designed to achieve a substantial reduction in the energy bill and RTGs peak electrical demand using a demand shaving strategy. Typically, the GA controller will work to charge or discharge the storage device during the lower or higher electric energy cost rate and demand periods, respectively. The electric energy cost is $90 £ / \mathrm{MWh}$ at Port of Felixstowe for the daytime from 7:00 am until midnight and $60 £ / M W h$ for the rest of the day. Based on the daily demand and cost analysis in the literature $[7,13,31]$ in addition to data provided by the port team, the annual electricity bill for two RTGs network is around $£ 20,442$.In Table 5, the annual electricity bill saving has been computed based on the cost of the annual average energy demand, and the average of the energy moved from high to low energy tariff periods. The GA controller with accurate forecast model can reduce the annual cost by around $7.12 \%$ compared to $5.47 \%$ for the set-point controller. The energy cost saving results, in Table 5, show, that the accurate forecasts improved the energy storage performance and increase the energy cost saving compared to control models with inaccurate 
forecasts on average by $1.21 \%$. In GA controller, the percentage of annual electric energy cost savings increased to $7.12 \%$ from $5.91 \%$. Although, the GA is computationally expensive, and it is required large data to train and validate the model. However, the GA model, is an off-line controller, and does not rely on extra communication and monitoring infrastructure to control the ESS [37,38] compared to other methods used in the literature. Hence, they can reduce or eliminate the required costs for the communications infrastructure, if the ESS schedule control can provide sufficient robustness to increase the storage benefit.

As presented in Figure 8 and Table 5, the proposed GA optimal controller outperforms the benchmark, setpoint controller. Due to the less predictable demand behaviour and the peak demand distributed over the 24 hours a day, the set-point controller usually depletes the stored energy quickly at insignificant peak points. The substation is then forced to feed the network of cranes. The resulting improvement in the optimal controllers is due to penalties the high energy costs and peak demand. The control algorithms using the accurate forecasts and historical data, increases the percentage of selected significant peak points over the day, this will increase the robustness of the ESS control plan.

Table 5. The percentage of annual electric energy cost saving to the annual electricity energy bill.

\begin{tabular}{ccc}
\hline Controller & \multicolumn{2}{c}{ Percentage of cost saving } \\
\cline { 2 - 3 } & Accurate & Inaccurate \\
\hline Set-point & $5.47 \%$ & $5.47 \%$ \\
SMPC & $7.12 \%$ & $5.91 \%$ \\
\hline
\end{tabular}

\section{Conclusions}

This paper has introduced an off-line control algorithm based on day-ahead RTGs demand forecast for a storage device in port network to reduce energy costs and RTGs peak demand. The off-line control scheme is a stochastic optimisation model based on a genetic algorithm. This control approach does not rely on perfect forecast and uses an ensemble of the day-ahead forecasts to run the ESS control model. Furthermore, as an offline controller, the model minimises the need for real-time communication and monitoring systems. The analysis results showed a significant peak reduction for the RTG crane demand for a specific case study by $28.7 \%$ for the GA control model and $23.9 \%$ for a set-point controller. The control approaches presented in the work have established the ability to which an ESS controller model can be an effective solution without including real-time solutions. The methodology of the GA control approach incorporates a novel aspect to control the ESS connected to network of cranes for the electric energy bill savings and RTGs peak demand reduction. The implementation of the stochastic optimal controller for ESS, improving the GA model by minimising the computational cost of the feasibility test and using an Active Front End (AFE) as a bi-directional converter in a network of RTG cranes is part of future work. The performance evaluation results for the control strategies are driven by the given demand data used throughout the paper.

\section{ACKNOWLEDGMENTS}

We would like to acknowledge the support of to the port of Felixstowe staff during collecting the data and providing all technical information. 


\section{References}

1. World Shipping Council. Trade Statistics [Online]. Available: http://www.worldshipping.org/about-the-industry/global-trade/tradestatistics. [Accessed 25 2018].

2. International Maritime Organisation. Statistical Resources - UNCTAD Review of Maritime Transport [Online]. Available: http://unctad.org/en/PublicationsLibrary/rmt2016_en.pdf. [Accessed 25 2018].

3. Yu, H.; Ge, Y.; Chen, J.; Luo, L.; Tan, C.; Liu, D. $\mathrm{CO}_{2}$ emission evaluation of yard tractors during loading at container terminals. Transportation Reserch Part D 2017, 53, 17-36.

4. Mach, J.; Proano, E.; Brown, K. Impacts of electric rubber-tired gantries on green port performance. Research in Transportation Business \& Management 2013, 8, 67-76.

5. Pietrosanti, S.; Holderbaum, W.; Becerra, V. Optimal Power Management Strategy for Energy Storage with Stochastic Loads. Energies 2016, 9, 175.

6. Alasali, F.; Haben, S.; Becerra, V.; Holderbaum, W. Day-ahead industrial load forecasting for electric RTG cranes. Journal of Modern Power Systems and Clean Energy 2018, 6, 223-234.

7. Alasali, F.; Haben, S.; Becerra, V.; and Holderbaum, W. Optimal Energy Management and MPC Strategies for Electrified RTG Cranes with Energy Storage System. Energies 2017, 10, DOI:10.3390/en10101598.

8. Zhao, N.; Schofield, N.; Niu, W. Energy Storage System for a Port Crane Hybrid Power-Train. IEEE Trans. Transp. Electrification 2016, 2, 480-492.

9. Kim, S.; Sul, S. Control of Rubber Tyred Gantry Crane With Energy Storage Based on Supercapacitor Bank. IEEE Transactions on Power Electronics 2006, 21, 5.

10. Flynn, M.; Mcmullen, P.; Solis, O. Saving energy using flywheels. IEEE Industry Applications Magazine 2008, 14 , issue: 6.

11. Niu, W.; Huang, X.; Yuan, F.; Schofield, N.; Xu, L.; Chu, J.; GU, W. Gu, Sizing of Energy System of a Hybrid Lithium Battery RTG Crane. IEEE Trans. Power Electron 2017, 32, 7837-7844.

12. Hellendoorn, H.; Mulder, S.; Schutter, B. Hybrid Control of Container Cranes. IFAC Proceedings Volumes 2011, 44, 9697-9702.

13. Alasali, F.; Haben, S.; Holderbaum, W. Energy management systems for a network of electrified cranes with energy storage. Electrical Power and Energy Systems 2019, 106, 210-222.

14. Rahmani-Andebili, M. and Fotuhi-Firuzabad, M. An Adaptive Approach for PEVs Charging Management and Reconfiguration of Electrical Distribution System Penetrated by Renewables. IEEE Transactions on Industrial Informatics. 2018, Vol. 14, NO. 5.

15. Pena-Bello, A.; Burer, M.; Patel, M.; Parra, D. Optimizing PV and grid charging in combined applications to improve the profitability of residential batteries. Journal of Energy Storage 2017, 13, 58-72.

16. Mohamed, F.; Koivo, H. Online management genetic algorithms of microgrid for residential application 2012, Energy Conversion and Management, 64, 562-568.

17. Rowe, M.; Yunusov, T.; Haben, S.; Holderbaum, W. and Potter, B. The Real-Time Optimisation of DNO Owned Storage Devices on the LV Network for Peak Reduction. Energies 2014, 7, 3537-3560.

18. Port of Felixstowe. Ship2shore magazine No 29. [Online]. Available: https://www.portoffelixstowe .co.uk/press/, [Accessed 20 5 2018].

19. Pandey, H.; Chaudhary, A.; Mehrotra, D. A comparative review of approaches to prevent premature convergence in GA. Applied soft computing 2014, 24, 1047-1077.

20. Ma, Y.; Zhang, C. Quick convergence of genetic algorithm for QoS-driven web service selection. Computer networks 2008, 52,10931104.

21. A. Crossland, D. Jones and N. Wade. Planning the location and rating of distributed energy storage in LV network using a gentic algorithm with simulated annealing. Electrical power and energy systems 2014, 59, 103-110.

22. Lee, H.; Shin, H.; Chae, J. Path Planning for Mobile Agents Using a Genetic Algorithm with a Direction Guided Factor. Electronics 2018, 7, 212; doi:10.3390/electronics7100212.

23. Cho, S.; Yan, S. Optimal power assignment of energy stoarge systems to improve the energy storage efficiency for frequency regulation. Energies 2017, 10, doi:10.3390/en10122092.

24. Cho, S.; Yan, S. Selection of Genetic Algorithm Parameters for Backcalculation of Pavement Moduli. International Journal of Pavement Engineering 2004, 5, 81-90.

25. Pongcharoen, P.; Hicks, C.; Braiden, P.; Stewardson, D. Determining optimum gentic algorithm parameters for scheduling the manufacturing and assembly complex product. International journal of production economics 2002, 78, 311-322.

26. Grefenstette, J. Optimization of control parameters for genetic algorithms. IEEE transactions on systems and cybernetics $1986,16$. 
27. Weron, R. and Ziel, F. Electricity price forecasting. Handbook Of energy economics, U.Soytas \& R.Sari (eds.), 2018. http://www.im.pwr.wroc.pl/ hugo/RePEc/wuu/wpaper/HSC_18_08.pdf

28. Hong-lei, W.; Wei, D.; Jain-Xin, C. The Dynamic Power Control Technology for the High Power Lithium Battery Hybrid RubberTired Gantry (RTG) Crane. IEEE Transactions on Industrial Electronic 2019, 66, 132-140.

29. Vahle. Port Technology [Online]. Available: https://www.vahleinc.com/port-technology.html, [Accessed 255 2018].

30. Vahle. Electrification of RTG cranes [Online]. Available:https://vahle.com/fileadmin/user_upload/ pdf/Broschueren/englisch/ VAHLE_Port_eRTG.pdf [Accessed 255 2018].

31. Papaioannou, V.; Pietrosanti, S.; Holderbaum, W.; Becerra, V.; Mayer, R. Analysis of Energy Usage for RTG Cranes. Energy 2017, $125,337-344$.

32. Zhang, Y.; Zhang, T.; Wang, R.; Liu, Y.; Guo, B. Optimal operation of a smart residential microgrid based on model predictive control by considering uncertainties and storage impacts. Solar Energy 2015, 122, 1052-1065.

33. Ji, Z.; Huang, X.; Xu, C.; Sun, H. Accelerated Model Predictive Control for Electric Vehicle Integrated Microgrid Energy Management: A Hybrid Robust and Stochastic Approach. Energies 2016, 9, 973.

34. Yunusov, T.; Haben, S.; Lee, T.; Ziel, F.; Holderbaum, W.; Potter, B. Evaluating the effectiveness of storage control in reducing peak demand on low voltage feeders. CIRED 2017, Glasgow, UK, PP. 1-5.

35. Rahmani-Andebili, M. Stochastic, adaptive, and dynamic control of energy storage systems integrated with renewable energy sources for power loss minimization. Renewable energy 2017, 113, 1462-1471.

36. Rahmani-Andebili, M. Dynamic and adaptive reconfiguration ofelectrical distribution system including renewables applying stochastic model predictive control. IET Generation, Transmission \& Distribution 2017, Vol. 11, pp. 3912-3921.

37. Rowe, M.; Yunusov, T.; Haben, S.; Holderbaum, W.; Potter, B. A peak reduction scheduling algorithm for storage devices on the low voltage network. IEEE Transactions on Smart Grid 2014. 5, 4.

38. Rahmani-Andebili, M. Chapter 9: Cooperative Distributed Energy Scheduling in Microgrids," Electric Distribution Network Management and Control, Springer, pp. 235-254, Apr. 2018. 